\title{
EXTRACTION OF GASTRIC MYOELECTRIC ACTIVITY FROM FINGER PHOTOPLETHYSMOGRAPHIC SIGNAL
}

\author{
S. Mohamed Yacin $^{1}$, M. Manivannan ${ }^{2}$, V. Srinivasa Chakravarthy ${ }^{3}$ \\ ${ }^{1,2}$ Biomedical Engineering Group, Department of Applied Mechanics, Indian Institute of Technology Madras \\ ${ }^{3}$ Computational Neuroscience, Department of Biotechnology, Indian Institute of Technology Madras \\ Chennai - 600036, Tamilnadu, \\ India \\ 1s_yacin@yahoo.co.in, ${ }^{2}$ mani@iitm.ac.in, ${ }^{3}$ svchakra@ee.iitm.ac.in
}

\begin{abstract}
This paper is an experimental study to examine the possibility of extracting gastric myoelectric activity (GMA) from photoplethysmographic (PPG) signals. Diagnosing GMA is a clinically challenging task because of its invasive/cumbersome methods. It is known that the PPG consists of information related to heart rate, respiratory rate and phenomena. Here we take this thread further and see whether GMA can be extracted from PPG in a simpler way and without discomfort to the patients. Since PPG and GMA signals are nonstationary, we choose discrete wavelet transform (DWT) to separate the different frequency components. PPG and Electrogastrog-ram (EGG, a method of measuring GMA) signals were acquired simultaneously at the rate of $100 \mathrm{~Hz}$ from 8 healthy subjects for 30 minutes duration in fasting and postprandial states. Both the signals were decomposed using DWT up to the frequency range $(0-0.1) \mathrm{Hz}$. A lower frequency oscillation $(\approx 0.05 \mathrm{~Hz})$ called slow wave was extracted from PPG signal which looks similar to the slow wave of GMA in both shape and frequency. Normalized cross-correlation technique was used for comparing the two signals. Cross-correlation values were found to be high $(\mathrm{R} \geq 0.73, \mathrm{R}=1.0$ indicates exact agreement) for all subjects without any significant change between fasting and postprandial states. The results suggest that there is a possibility of extracting gastric related information from PPG signals using appropriate signal processing techniques. In future this novel technique could be used as a diagnostic tool for gastrointestinal system disorders.
\end{abstract}

\section{KEY WORDS}

Noninvasive measurement, data and signal acquisition, blood volume pulse, gastric myoelectric activity

\section{Introduction}

Photoplethysmography (PPG) is a low cost, simple, noninvasive, electro-optic method which measures the volumetric changes in blood vessels that mainly occur in arteries and arterioles [1]. PPG gained popularity because it is easy to acquire and contains numerous clinical parameters such as heart rate, respiratory induced intensity variations (RIIV) [2],[3],[4], oxygen saturation level in blood (called as pulse oximeter) [5]. In PPG, an infrared beam traveling through the fingertip is absorbed by pulsatile arterial blood, venous blood and other absorbing tissues such as skin pigmentation and bone, and the transmitted or reflected beam is detected by a photo detector [6],[7]. PPG signal mainly consists of two components, pulsatile component due to the arterial blood (AC component) and the stationary part (DC component) due to absorbance of venous blood, the fixed quantity of arterial blood, and other stationary components like skin pigmentation [7]. PPG also is used to evaluate autonomous nervous system (ANS) based on peak-topeak variability [8]. It has been found that the PPG signal is multi-component in nature and has nearly five different frequency components in the interval $(0.007-1.5) \mathrm{Hz}$ [9]. These frequency components may be related to heart rhythm, respiratory rate, blood pressure control, thermoregulation, and autonomous nervous system (ANS) [10]. Sources of these components present in PPG signal are not completely examined because of interconnected nature of the cardiovascular system, specifically in the skin microcirculation where regulatory processes of both central and local origin are involved [14].

Our hypothesis is that since human circulatory system constitutes many interacting subsystems, rhythm changes in one subsystem could possibly manifest in the processes some of the other subsystems. Therefore, hemodynamics in any separate subsystem could be observed by hemodynamic interactions throughout the whole system because its closed-loop nature [11]. Based on this, we are trying to extract gastrointestinal (GI) system related GMA from radial artery PPG signal. Diagnosing the internal visceral organs such as stomach, kidney and its disorders by non-invasive PPG is a clinically challenging task. A quantitative report of abdominal PPG signals have been investigated invasively and showed that the PPG can also be used to measure blood volume change in abdominal organs [12]. It was also stated that the peripheral blood flow dynamics changes due to change in blood supply to 
the smooth muscles of the stomach during digestion [13]. Wavelet analysis of blood flow signal measured by PPG and laser Doppler flow meter was studied. It was shown that the rhythmic oscillation in the frequency band $(0.04-$ $0.1) \mathrm{Hz}$ may be due to myogenic activity of smooth muscles or neurogenic activity [14], [15]. This observation supports our hypothesis that GMA-related information may also be present in PPG and it can be extracted using appropriate signal processing techniques.

Human stomach is an enlarged, muscular sac-like organ of the alimentary canal and the principal organ of digestion. Its motor functions include accommodation of ingested food, grinding food chunks, mixing of secretory gastric juice into particles, and delivery of food chyme into the duodenum. In order to accomplish the whole digestive process of the stomach, from mixing, stirring, agitating, propelling and to emptying, a spatiotemporal activation pattern is formed [16]. This pattern is called gastric myoelectrical activity (GMA) which originates from the pacemaker located in proximal body of the stomach. It manifests the continuously rhythmic change in the membrane potential and thereby propagates to the distal antrum with a regular frequency of about 3 cycles per minute $(0.05 \mathrm{~Hz})$. Normal GMA was defined as a frequency between 2 and $4 \mathrm{cpm}$. It is believed that the interstitial cells of Cajal (ICC) of enteric nervous system (ENS) generate the rhythmic depolarizations of the gastric slow wave. Additional depolarizations provided by neurohumoral stimulation are the triggers for phasic gastric contractions which follow the spread of the electrical slow waves and are peristaltic. Thus, gastric electrical slow waves control the maximal frequency and the direction of contractions in the distal stomach [17]. Recording of this GMA is called electrogastrography (EGG) and it can be measured by positioning surface electrodes noninvasively over the abdominal skin. EGG measurement shows a slow wave pattern with reasonable accuracy the overall GMA function [18],[19].

The relationship between PPG signals and cardiopulmonary system parameters has been found widely in the literature. But, very few reports have been examined concerning the relationship between PPG and EGG signals. The aim of the current study is to examine the possibility of extracting GMA related information from PPG signal, considering that GMA is a myoelectric activity triggered by ENS. The PPG and EGG are nonstationary signals in nature by means of its frequency, magnitude and shape of the wave. So the selected signal processing approach to be selected must suitable for nonstationary signals, which sidelines many traditionally followed filtering techniques [22]. Discrete Wavelet Transform (DWT) is designed for nonstationary signals because it considers the term "scale" into the transform, which can give good time-frequency resolution. A compressed wavelet is considered for processing highfrequency details and an elongated wavelet for processing lower frequency patterns [20]. The DWT method was utilized for multi-level decomposition of PPG and EGG signals of 8 healthy subjects in fasting and postprandial states. An established way of comparing two signals is cross-correlation. Many fields like speech processing and image processing is using cross-correlation. Since crosscorrelation has been used to assess the similarity of PPG signals acquired from ears, thumbs and toes [21]. But from our own knowledge, cross-correlation never used to compare PPG and EGG signals after performing DWT multi-level decomposition between different individuals under fasting and postprandial states. This paper examines whether GMA can be extracted from PPG signal and analyses the level of correlation.

\section{Materials and methods}

\subsection{Subjects}

This study was executed with 8 healthy non-habitual smoking and non-sports male subjects without disorders and symptoms of gastrointestinal, cardiovascular or any other diseases. The volunteers were recruited from the student community of our institute. Subjects mean age was $22.0 \pm 2.7(\mathrm{SD})$ years in the range of 20 and 28 years and the mean body mass index (BMI) was $22.3 \pm 1.7$ (SD) (range 19.7-25.3). This study was approved by our institute ethics committee and all the subjects were given informed consent before data recording.

\subsection{Data acquisition and hard ware}

We acquired the PPG signal from the index finger using a reflection type infrared sensor (SS4LA, Biopac Systems, Inc, USA). Volunteers were informed to observe silence and to keep relatively still during data recording time to minimize motion artifacts. EGG signals are measured by Ag-Agcl electrodes (SS2L, Biopac Systems, Inc, USA) placed over the abdominal surface. The skin area in the abdominal surface was cleaned with sandy skin prepping paste to reduce the skin resistance and to minimize skin electrode motion artifacts. Three disposable surface electrodes filled with electrodes jelly were placed over on the abdominal skin. Out of three, two active electrodes were placed below the left costal margin and in between the xyphoid process and umbilicus. The third one considered as reference electrode was positioned in the right upper quadrant. Real time recording unit MP 35 (Biopac Systems, Inc, USA) is used here for data acquisition.

After allowing the subject to rest in a supine position for 15 minutes in order to maintain stable the heart beat and respiration, PPG and EGG signals were recorded in a following manner. Fasting data were recorded for 30 minutes in the supine position after 5 hours of fasting. Then the subjects were allowed to take meal (comprised of limited rice, fruit slices and one cup of water) in a sitting position and then again assume supine position. 
Data were also collected in postprandial condition immediately after meal for more than 30 minutes.

During data recording procedure, all the subjects were asked to maintain the breathing rate at more than 10 cycles per minute (or at 12 cycles per minute $=0.2 \mathrm{~Hz}$ ) and to the extent possible, kept quiet and remain in the same position. Temperature was regulated at $25 \pm 1^{\circ} \mathrm{C}$ in the data recording room. During acquisition the gain was adjusted to 2000 for PPG signal and it was 5000 for EGG signal. Both the signals were acquired at the sampling rate of 100 samples per second. The major movement artefacts are found by direct visual inspection of the waveform. Abnormally large positive or negative peaks in the tracing were identified as movement artifact by direct visual analysis; the same was removed using a separate program. A portion of the signals recorded for the same subject during fasting and postprandial states are shown in fig 1 and fig 2. A dedicated personal computer (PC) was utilized for acquiring, display, storage and analysis of PPG and EGG data.

\subsection{Discrete wavelet transform}

The wavelet analysis talks about approximations and details. The approximations are defined as the high-scale, low-frequency contents and the details are defined as the low-scale, high-frequency contents present in the signal. DWT decomposes the signal into coarse approximation and detail coefficients as shown in Fig. 3 at different frequency subbands with different resolutions. DWT can be mentioned by means of a low-pass filter $h$, which satisfies the standard quadrature mirror filter condition:

$$
H(z) H\left(z^{-1}\right)+H(-z) H\left(-z^{-1}\right)=1
$$

where $H(z)$ denotes filter $\mathrm{h}$ z-transform and its complementa high-pass filter can be given as,

$$
G(z)=z H\left(-z^{-1}\right)
$$

A series of filters with increasing length (indexed by i) can be derived.

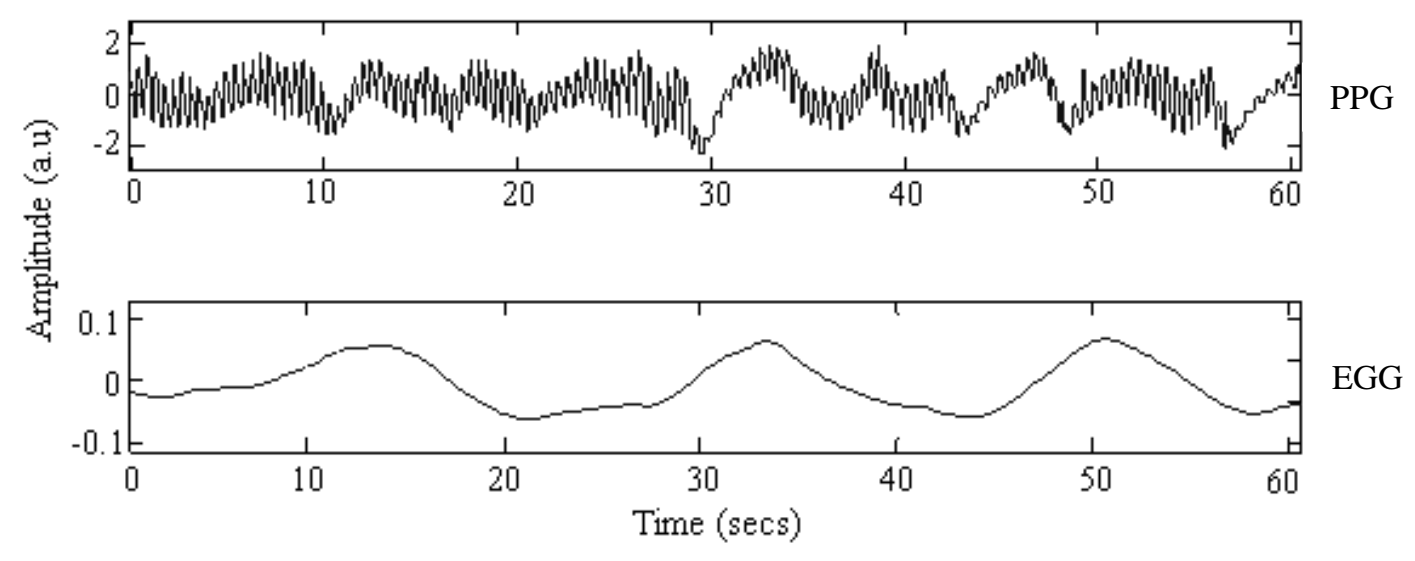

Figure.1. PPG and EGG signals recorded in fasting state

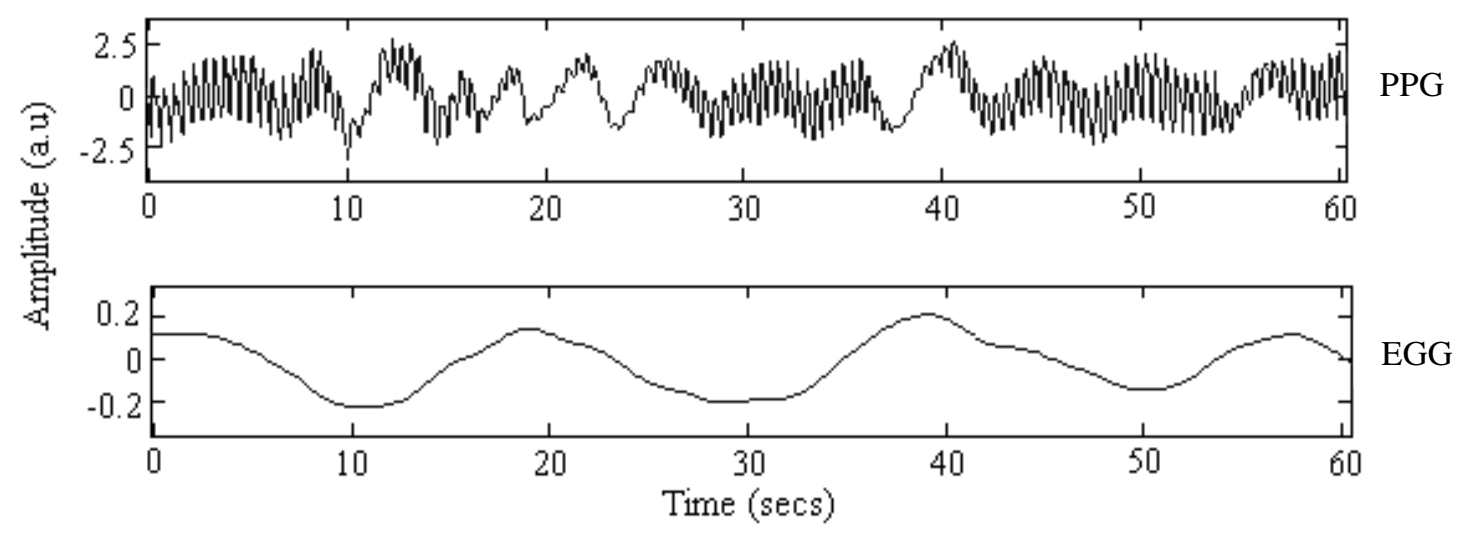

Figure.2. PPG and EGG signals recorded in postprandial state 


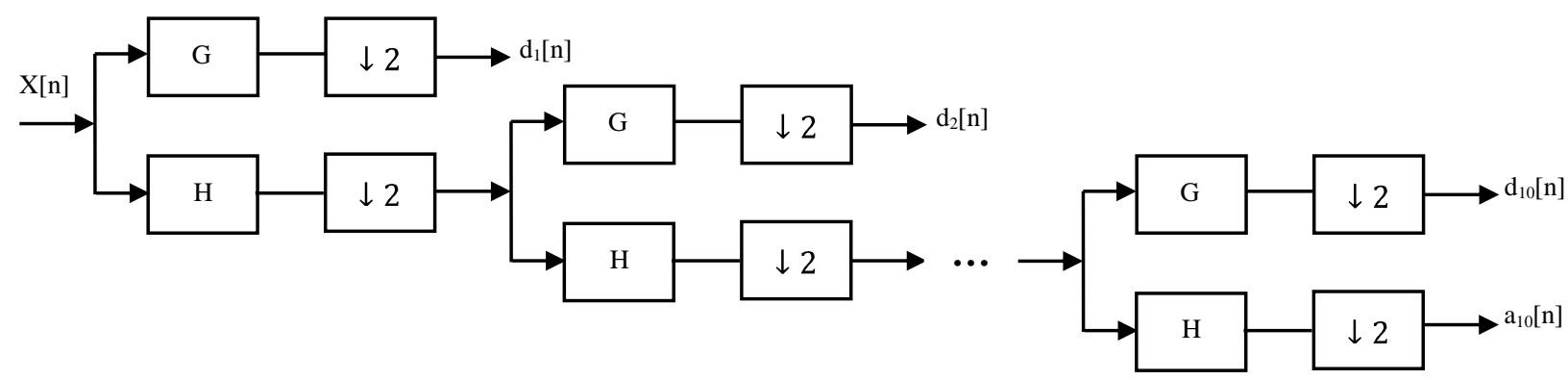

Figure.3. Discrete wavelet transform multi-level decomposing process tree

$$
\begin{aligned}
& H_{i+1}(z)=H\left(z^{2^{i}}\right) H_{i}(z), \\
& G_{i+1}(z)=G\left(z^{2^{i}}\right) H_{i}(z), \quad i=0, \ldots I-1
\end{aligned}
$$

with the initial condition $H_{0}(z)=1$. It is expressed as a two-scale relation in time domain

$$
\begin{aligned}
& h_{i+1}(k)=[h]_{\uparrow 2^{i}} * h_{i}(k), \\
& g_{i+1}(k)=[g]_{\uparrow 2^{i}} * h_{i}(k),
\end{aligned}
$$

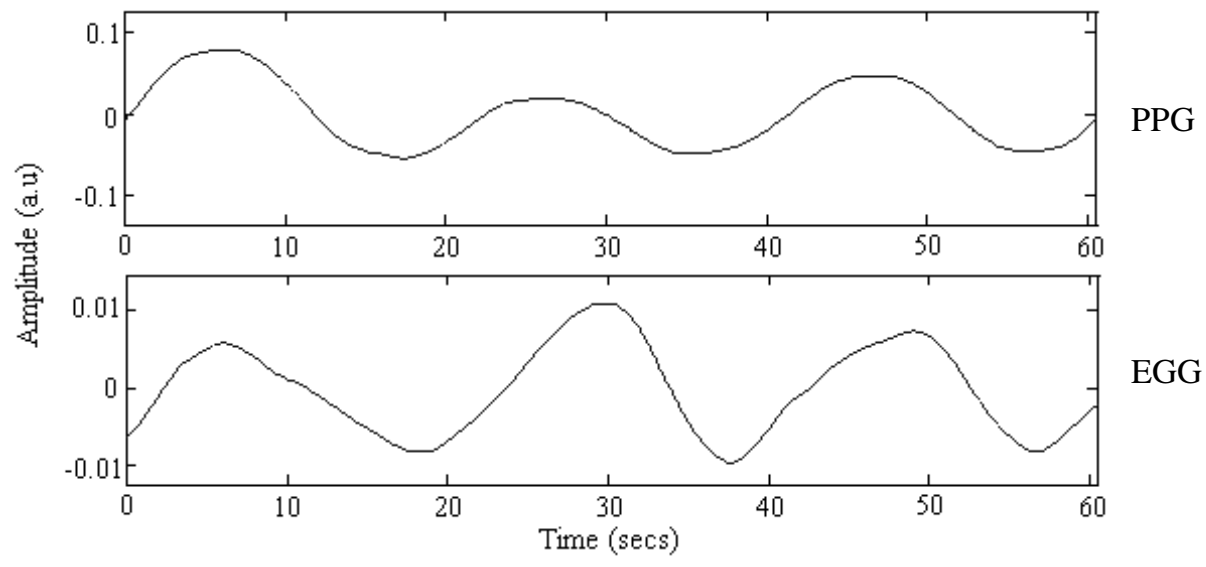

Figure. 4. Slow waves reconstructed from $10^{\text {th }}$ level approximation matrices in fasting state

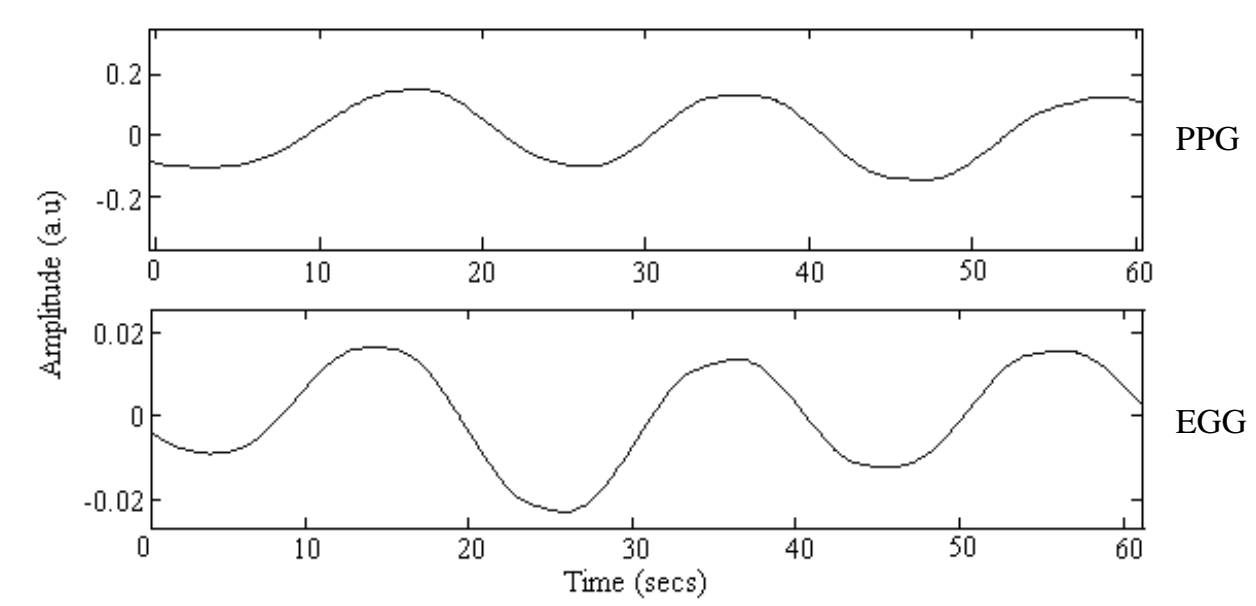

Figure.5. Slow waves reconstructed from $10^{\text {th }}$ level approximation matrices in postprandial state where the subscript $[\cdot \leftarrow] \uparrow j$ indicates the up-sampling by a factor of $\mathrm{j}$, and $\mathrm{k}$ is the equally sampled discrete time. DWT has two sets of functions, one is scaling function and the other is wavelet function, these are associated with low-pass and high-pass filters, respectively. The normalized wavelet and scale basis functions $\varphi_{i, l}(k), \psi_{i, l}(k)$ can be defined as,

$$
\begin{aligned}
& \varphi_{i, l}(k)=2^{i / 2} h_{i}\left(k-2^{i} l\right), \\
& \psi_{i, l}(k)=2^{i / 2} g_{i}\left(k-2^{i} l\right),
\end{aligned}
$$


where factor $2^{i / 2}$ is an inner product normalization, and $\mathrm{i}$ and 1 are the scale parameter and the translation parameter, respectively. The DWT can be described as

$$
\begin{aligned}
& s_{i}(l)=x(k) * \varphi_{i, l}(k), \\
& d_{i}(l)=x(k) * \psi_{i, l}(k),
\end{aligned}
$$

where $s_{i}(l)$, the approximation coefficients and $d_{i}(l)$ the detail coefficients respectively at resolution i, [22], [23], [24].

The high pass filter in each level produces details information; $d[n]$, while the low pass filter associated with scaling function produces coarse approximations, $\mathrm{a}[\mathrm{n}]$. At each decomposition level the filter frequency range becomes half of the frequency band and produce signals spanning only half the frequency band. This principle doubles the frequency resolution as the uncertainty in frequency is reduced by half. According to Nyquist's criterion if the original signal has highest frequency of $\omega$, which needs a sampling frequency of $2 \omega$ radians, then it now has a highest frequency of $\omega / 2$ radians [27]. Now the signal can be sampled at a frequency of $\omega$ radians thus omitting half the samples without loss of information. Such kind of decimation by 2 halves the time resolution as the complete signal is now represented by half of the samples. As explained, since the half-band low-pass filtering removes half of the frequencies and thus halves the resolution, the decimation by 2 doubles the scale. According to this principle, the time resolution of the signal at high frequencies becomes arbitrarily good, since the frequency resolution at low frequencies becomes arbitrarily good[27]. For this study, decomposition of the signals was performed using Daubechies mother wavelet [26] of order ('db3'). $10^{\text {th }}$ level approximation coefficients matrices, which correspond to the frequency range $(0-0.1) \mathrm{Hz}$ is taken for further analysis. A signal, called slow wave is reconstructed from $10^{\text {th }}$ level approximation coefficients matrices of PPG and EGG signals and are shown in Fig. 4 and Fig. 5, respectively. The horizontal axis represents time in seconds and the vertical axis represents normalized amplitude of the signal. All these signal processing techniques were done in MATLAB 7.0 release 14.1 (The MathWorks co, USA, MATLAB ${ }^{\circledR}$ version 7.2).

\subsection{Cross-Correlation analysis}

Cross-correlation between two time series measures the degree of similarity between the signals [28]. This gives a measure of the degree of relatedness between two signals quantitatively, normally from two different recording sites which are progressively shifted in time with respect to each other. Consider two series $x_{i}$ and $y_{i}$ are the slow wave of PPG and EGG, where $\mathrm{N}$ is the number of samples and $i=0,1,2, \ldots \mathrm{N}-1$ [29]. Considering zero time lag and the normalized cross-correlation function is defined as,

$$
R=\frac{\sum x_{i} y_{i}}{\left(\sum x_{i}^{2}\right)^{1 / 2}\left(\sum y_{i}^{2}\right)^{1 / 2}}
$$

Equation (11) calculates the degree of similarity in shape between two curves as a scalar between 0 and 1 , which is analogous to the dot product of two vectors. If the two signals with exactly the similar shape will give a crosscorrelation value of 1.0. Cross-correlation results do not change while changing the amplitude of the curve only when there is no change in its shape. R-values are most sensitive to similarities and differences in timing; when timing is similar, they are also sensitive to similarities and differences in shape. In this work cross-correlation analysis was done to compare slow waves of PPG and EGG for different body conditions.

\section{Results and discussion}

Many experimental results are available on PPG lower frequency oscillations that reveal information about cardiopulmonary parameters with different level of accuracies. But very few researchers paid attention over the GMA interaction which is a part of the physiological system. Here we have chosen DWT for feature extraction because of its high resolution. The DWT coefficients from the $10^{\text {th }}$ approximation level which corresponds to the frequency of $(0-0.1) \mathrm{Hz}$ were extracted from both PPG and EGG separately and cross-correlation analysis were performed for all the healthy subjects. A filtered EGG slow wave was reconstructed from DWT coefficients matrices of $10^{\text {th }}$ approximation level because most of the artifacts are removed by the high pass filters (details). In PPG DWT decomposition, the expected high frequency components such as heart rate $(\approx 1 \mathrm{~Hz})$ and respiratory rate $(\approx 0.3 \mathrm{~Hz})$ information were also removed using high pass filters (details) and the slow wave was taken for analysis which are shown in fig.4 for fasting state and fig.5 for postprandial state for same subject. The difference in amplitudes of slow waves of PPG and EGG (in volts and in micro volts) may be due to their signal strengths in acquisition. Results from only one subject are displayed here; almost similar results are obtained for all other subjects.

The cross-correlation analysis performed has given promising results of R-value were greater than 0.7 (95\% confidence level) for most of the subjects and is shown in Table I. This indicated that the slow wave patterns of PPG and EGG for an individual subject are consistent in different body conditions. This supports that PPG signal may have information about GMA because of physiological nature of the system and needs to be confirmed by further research. Extracting GMA using DWT will be a useful method for any given subject at different time points, such as fasting and postprandial states. While this research provides a new, simple and alternative method for the extraction of GMA, it should also be noted that the accuracy of this method is connected with the PPG recording and selection of mother 
wavelet. So it is very important to note that, during data recording, maximum effort should be made to have highest possible signal-to-noise ratio, appropriate placement of PPG sensor, minimization or elimination of motion artifacts and environmental conditions. Though some studies investigate the variability of PPG signal in ANS [24], the function of enteric nervous system (ENS), which is considered as a part of ANS, is not well explored. GMA amplitude is higher in the postprandial state and was observed as increase in EGG signal power (square of the amplitude per $\mathrm{Hz}$ ), which is wellestablished and proved [17]. This may be because of the fact that the increase in EGG power is the effect of increase in splanchnic circulation blood supply to the gastric muscle for strong muscle contraction during digestion and absorption of nutrients. The rhythm corresponding to these large blood volume changes in the gut, seems to manifest itself in the finger PPG. We are not considering the increase in power of the signal here which is beyond the scope of this work because our objective is to extract the GMA information from PPG signal. In this preliminary work it is revealed that there is a significant correlation between PPG and EGG (Slow waves) in healthy humans.

Table I Comparison of Cross-correlation results

\begin{tabular}{|c|c|c|}
\hline \multirow{2}{*}{$\begin{array}{l}\text { Subject } \\
\text { Number }\end{array}$} & \multicolumn{2}{|c|}{ Normalized cross-correlation (R) values } \\
\hline & $\begin{array}{c}\text { R-value in fasting } \\
\text { state }\end{array}$ & $\begin{array}{l}\text { R-value in postprandial } \\
\text { state }\end{array}$ \\
\hline 1 & $0.72 \pm 0.01$ & $0.73 \pm 0.02$ \\
\hline 2 & $0.71 \pm 0.02$ & $0.72 \pm 0.13$ \\
\hline 3 & $0.73 \pm 0.11$ & $0.74 \pm 0.02$ \\
\hline 4 & $0.79 \pm 0.06$ & $0.79 \pm 0.13$ \\
\hline 5 & $0.71 \pm 0.03$ & $0.73 \pm 0.07$ \\
\hline 6 & $0.67 \pm 0.12$ & $0.68 \pm 0.03$ \\
\hline 7 & $0.71 \pm 0.01$ & $0.72 \pm 0.12$ \\
\hline 8 & $0.72 \pm 0.08$ & $0.73 \pm 0.02$ \\
\hline
\end{tabular}

\section{Conclusion}

Feature extraction of clinically important parameters from PPG is gaining popularity because of its low cost, noninvasiveness and ease acquisition. Estimation of GMA from EGG is difficult because of its poor signal-to-noise ratio and discomfort to the patients. In this study DWT is used for the extraction of GMA slow wave from PPG by decomposing the signal into details and approximation coefficients. The use of a DWT cross-correlation analysis allowed a closer investigation of the lower frequency oscillations of PPG and its intermittent behavior during fasting and postprandial conditions. This study indicates that there is a good correlation $(\mathrm{R} \geq 0.73)$ between the slow wave of PPG with EGG. Results of this study primarily indicate a development of PPG technology in diagnosing GI system. Extending this methodology to gastric pathology cases like stomach ulcer may provide further corroborative insights on PPG usage, which can be performed as a future work. The peripheral blood volume signal, measured by PPG, reflects the dynamics of the entire cardiovascular system. The measured volume is modulated by the heart and respiratory functions as well as by local mechanisms of resistance control. This study is limited to a small group of healthy volunteers and needs to be extended to larger groups and also include diseased states.

In conclusion, obtaining GMA information in PPG signal might offer new insights into clinical diagnosis. The stated results show that the proposed method can make an effective interpretation. Our future efforts will be directed towards actually reconstructing EGG from PPG. If this attempts results in success, we will have an elegant noninvasive clinical tool for monitoring gastric myoelectrical activity in health and disease.

\section{Acknowledgements}

We wish to thank all the volunteers for sparing their valuable time and effort to make this study successful.

\section{References}

[1] A V J. Challoner, Photoelectric plethysmography for estimating cutaneous blood flow. Non-Invasive Physiological Measurements (edited by Rolfe, P), vol 1, 1979, 125-51.

[2] A Johansson \& Oberg $\mathrm{P}^{\circ} \mathrm{A}$, Estimation of respiratory volumes from the photoplethysmographic signal. Part1: experimental results, Medical \& Biological Engineering \& Computing., vol 37,1999, 42-47.

[3] A Johansson \& Oberg $\mathrm{P}^{\circ} \mathrm{A}$, Estimation of respiratory volumes from the photoplethysmographic signal. Part 2: a model study, Medical \& Biological Engineering \& Computing, vol 37, 1999, 48-53.

[4] L. Nilsson, A. Johansson \& S. Kalman, "Monitoring of respiratory rate in postoperative care using a new photoplethysmographic technique", Journal of Clinical Monitoring ., vol.16, 2000, 309-315.

[5] J. Allen, "Photoplethysmography and its application in clinical physiological measurement", Physiological Meas., vol.28, 2007, 3, R1-R39.

[6] A. B. Hertzman \& C. R. Spielman, "Observations on the finger volume pulse recorded photoelectrically", Am. J. Physiol.,vol.119, 1937, 334-335.

[7] Yue-Der Lin, Wei-Ting Liu, Ching-Che Tsai, and Wen-Hsiu Chen, Coherence Analysis between Respiration and PPG Signal by Bivariate AR Model, World Academy of Science, Engineering and Technology, 53,2009, 847-852

[8] M Nitzan, A Babchenko , B Khanokh \& D Landau, The variability of the photoplethysmographic signal: a 
potential method for the evaluation of the autonomic nervous system, Physiological Measurement, vol 19, 1998, 93-102.

[9] B.W. Hyndman, R. I. Kitney \& B. McA. Sayers, Spontaneous rhythms in physiological control systems. Nature ,vol 233, 1971, 339-341.

[10] M Nitzan, S Turivnenko, A Milston, A Babchenko \& $\mathrm{Y}$ Mahler, Low-frequency variability in the blood volume pulse measured by photoplethysmography $J$. Biomedical Optics, vol 1, 1996, 223-9.

[11] Fuyou Liang \& Hao Liu, A Closed-Loop Lumped Parameter Computational Model for Human Cardiovascu-lar System, JSME International Journal, Series C, Vol 48, 2005, No:4, 484-493.

[12] P A Kyriacou, A Crerar-Gilber, R M Langford \& D $\mathrm{P}$ Jones, Electro-optical techniques for the investigation of photoplethysmographic signals in human abdominal organs, Journal of Physics: Conf. Series vol. 45, 2006, 232-238.

[13] E Clinton Texter, Small Intestinal Blood Flow, New Series, Vol.8, No.7, 1963, 587-613.

[14] A Stefanovska, M. Bra cǐ c, \& H. D. Kvernmo. Wavelet analysis of oscillations in the peripheral blood circulation measured by laser Doppler technique. IEEE Trans. Bio. Med. Eng., vol 46(10), 1999, 1230-1239.

[15] P Kvandal, S. A. Landsverk, A. Bernjak, U. Benko, A Stefanovska, H. D. Kvernmo, \& K. A. Kirkebøen. Low frequency oscillations of the laser Doppler perfusion signal in human skin. Microvasc. Res., vol. 72(3): 2006, 120-127.

[16] Fatma Dirgenali, Sadık Kara, S Sükrü Okkesim, Estimation of wavelet and short-time Fourier transform sonograms of normal and diabetic subjects' electrogastrogram, Computers in Biology and Medicine 36 (2006) 1289-1302.

[17] A.J. Smout, E.J. Van der Schee, What is measured in electrogastrography?, Dig. Dis. Sci.,1980, 179-187.

[18] H.P.Parkman, "Electrogastrography: a document prepared by the gastric section of the American Motility Society Clinical Testing Task force", Neurogastroenterol. Motil, vol.15, 2003, 89-102.

[19] W.C. Alvarez, The electrogastrogram and what it shows, JAMA, 1922, 116-119.

[20] Xinling Shi, Baodan Bai, Yufeng Zhang, Huahong Ma, Jianhua Chen, Extraction of mean frequency information from Doppler blood flow signals using a matching pursuit algorithm,Signal Processing 88 (2008) $2720-2730$

[21] J Allen \& A Murray, Similarity in bilateral photoplethysmographic peripheral pulse wave characteris-tics at the ears, thumbs and toes. Physiological Measurement,.vol 21, 2000a, 369-377.

[22] J.H. Li, H. Peng, Y.Y. Du, J.H. Li, W.H. Wang, Analysis of Doppler ultrasound blood flow signal basing on wavelet transform, J. Northeastern Univ. (Nat. Sci.) vol. 21 (5), 2000, 487-489.

[23] J.M.Girault, D.Kouame, A.Ouahabi, F. Patat, Microemboli detection: an ultrasound Doppler signal processing viewpoint, IEEE Trans. Biomed. Eng. 47 (2000) 1431-1439.

[24] Y. Zhang,Y.Wang, W.Wang, \& B. Liu, Doppler ultrasound signal denoising based on wavelet frames, IEEE Trans. Ultrason. Ferroelectr. Frequency Control 2001, 48, 709-716.

[25] J. Chen \& R.W. McCallum, Response of the electric activity in the Human stomach to water and a solid meal. Medical \& Biological Engineering \& Computing, vol 29, 1991, 351- 357.

[26] Daubechies, I., The wavelet transform, timefrequency localization and signal analysis. IEEE Trans. Inf. Theory, vol.36(5), 1990, 961-1005.

[27] Yue Yuan, Xiaoming Yu and Hongji Du, Power System Fault Data Compression Using the Wavelet Transform and Vector Quantification, International Conference on Power System Technology, 2006, 1-6.

[28] Y Mizuno-Matsumoto , S Tamura, Y Sato, Zoroofi RA, Yoshimine T, Kato A, Taniguchi M, Takeda M, Inouye $\mathrm{T}$, Tatsumi $\mathrm{H}$, Shimojo $\mathrm{S}$, Miyahara $\mathrm{H}$. Propagating process of epileptiform discharges using wavelet-cross-correlation analysis in MEG. Tohoku University Press, 1999a, 782-785.

[29] A L Tishya Wren, K. Patrick Doa, Susan A. Rethlefsena, Bitte Healy, Cross-correlation as a method for comparing dynamic electromyography signals during gait, Journal of Biomechanics 39,2006, 2714-2718

[30] K Nakajima ,T Tamura \& H Miike, Monitoring of heart and respiratory rates by photoplethysmography using a digital filtering technique, Medical Engineering Physics, vol 18,1996, 365-372.

[31] J N Cohn, Non-invasive pulse wave analysis for the early detection of vascular disease, Hypertension, vol 26, 1995, 503-508.

[32] Dieter Barschdorff \& Wei Zhang, Respiratory Rhythm Detection with Photoplethysmographic Methods, Proc. of the $16^{\text {th }}$ Annaul International conference of EMBS, IEEE Transactions on BME vol.2 , Baltimore, MD, USA, 1994, 912-913.

[33] Michio Watanabe, Yutaka Shimada, Shinya Sakaia, Naotoshi Shibahara, Harumi Matsuda, Katsumi Umeno, Hidetsugu Asanoi and Katsutoshi Terasawa, Effects of water ingestion on gastric electrical activity and heartrate variability in healthy human subjects, Journal of the Autonomic Nervous System 58,1996, 44-50. 